Research Square
Sactors Associated With
Abbott Japan LLC
Masashi Nishimura
Tokyo Shinagawa Hospital
Shinichiro Ota ( $\square$ shin.ohta0915@gmail.com )
Tokyo Shinagawa Hospital
Miwa Morikawa
Tokyo Shinagawa Hospital
Takashi Sato
Tokyo Shinagawa Hospital
Masahiro Shinoda
Tokyo Shinagawa Hospital
Naoki Ishii
Tokyo Shinagawa Hospital
Kenichi Kamachi
Tokyo Shinagawa Hospital
Masaharu Shinkai
Tokyo Shinagawa Hospital

\title{
Factors Associated with Low IgG Response to mRNA COVID-19 Vaccine BNT162b2
}

Satoshi Sugawa

Masashi Nishimura

Tokyo Shinagawa Hospital

Shinichiro Ota ( $\nabla$ shin.ohta0915@gmail.com )

Tokyo Shinagawa Hospital

Miwa Morikawa

Tokyo Shinagawa Hospital

Takashi Sato

Masahiro Shinoda

Tokyo Shinagawa Hospital

Tokyo Shinagawa Hospital

Tokyo Shinagawa Hospital

Tokyo Shinagawa Hospital

\section{Research Article}

Keywords: COVID-19, SARS-CoV-2, BNT162b2, immune response, receptor binding domain, ACE2, vaccination

Posted Date: November 17th, 2021

DOl: https://doi.org/10.21203/rs.3.rs-969684/v1

License: (9) This work is licensed under a Creative Commons Attribution 4.0 International License. Read Full License 


\section{Abstract}

Amid pandemic caused by severe acute respiratory syndrome coronavirus 2 (SARS-CoV-2), vaccination is hailed as one of the most effective preventive measures. An mRNA vaccine termed BNT162b2 showed satisfactory safety and efficacy in the clinical trials but several issues including variability in the individual immune response or determination of target antibody titre that exerts sufficient immune protection needs to be assessed for planning a more efficient vaccination strategy. By monitoring IgG titres before and two months after the initial administration of BNT162b2 in 655 healthcare workers by Abbott IgG II quant that detects IgG against the receptor-binding domain in S protein of SARS-CoV-2, we confirmed that hypertension, dyslipidaemia, chronic kidney disease and use of immune suppressant in addition to male gender, advanced age, and absence of previous infection were significantly associated with low antibody response to the vaccination.

\section{Introduction}

Vaccination against severe acute respiratory syndrome coronavirus 2 (SARS-CoV-2) is hailed as one of the most effective measures for the prevention of COVID-19, a disease caused by SARS-CoV-2. An mRNA vaccine termed as BNT162b2 which was developed by BioNTech and Pfizer and was designed to produce spike protein (S protein) of SARS-CoV-2 in human body to elicit antibody response, showed satisfactory safety and efficacy in the clinical trials [1]. The vaccination to the general population with this vaccine was initiated following the issuance of emergency use authorization from the food and drug administration (FDA) in the United States in December 2020, followed by several other countries including Japan. In Japan, fast-track approval was granted to the vaccine on 14 February 2021 and the Japanese government started the vaccination initially to the healthcare workers [2] followed by elder people and others.

In the phase 2 and 3 study of BNT162b2, the efficacy against the placebo was 95\% [1]. In consistent with this result, the efficacy reported from a nationwide mass vaccination study in Israel was $92 \%$ [3]. By using Abbott IgG II quant that detects IgG against receptor binding domain in S protein (hereinafter designated as "IgG (RBD)"), Naaber et al. reported, however, that the antibody responses were weaker and waning faster in elder and male populations, which suggested decreased efficacy of the vaccination in these populations [4]. By quantitative measurement of $\mathrm{IgG}$ antibodies against S1/S2 antigens of SARS-CoV-2, Pellini et al. also showed that people with advanced age and those of male gender were statistically associated with weaker antibody response after vaccination, whereas obesity and hypertension showed no statistically significant association [5]. The variability of individual antibody responses, however, suggests the existence of factors other than age or male gender that affect the immune response to the vaccination. If we could have better understandings about those factors, we would be able to improve the vaccination strategy basing on the individual background.

In addition to the variability of the antibody response mentioned above, there is an emerging concern on reduced efficacy of the vaccination against SARS-CoV-2 variants. Liu et al. reported that reduction of 
neutralizing activity in sera from subjects vaccinated with BNT162b2 was seen in the reaction with some variants of SARS-CoV-2, notably the B.1.617.1 [6]. Davis et al. also reported not only the B.1.617.1 but also B.1.617.2 (delta) and B.1.351 showed reduced neutralizing effect [7]. Pfizer announced a preliminary result showing third vaccine dose strongly boosted neutralizing activity against the variant delta [8], which suggests that the higher titre and matured affinity of the antibody would be one of the measures to overcome the emergence of the variants.

To effectively vaccinate the population under the above circumstances, we would need to quantitate the effectiveness of the vaccination with a high-throughput method and stratify groups of population necessary for the re-vaccination. Gobbi et al. compared antibody responses to BNT162b2 with IgG (RBD) and neutralizing IgG assays [9]. In this study, elevation of the neutralizing antibody defined by a binding activity to $50 \%$ or more of ACE2 was observed in more than $97.8 \%$ of the study subjects seven days after the second dose. Similarly, elevation of IgG (RBD) above 4,160 AU/mL was observed in more than $97.4 \%$ of the study subjects seven days after the second dose, so that the cut-off $4,160 \mathrm{AU} / \mathrm{mL}$ was considered equivalent to the neutralizing activity defined above. Garcia-Beltran et al. also reported that titre of IgG against receptor binding domain of SARS-CoV-2 detected by enzyme-linked immunosorbent assay (ELISA) was correlated with $50 \%$ neutralizing activity (NT50) with the correlation determination $\mathrm{R}^{2}$ of 0.78 [10]. Moreover, Muecksch et al. reported that correlation coefficient (R) between IgG (RBD) and NT50 with sera from subjects 3.5-8.5 weeks post PCR positivity was 0.8977 [11]. From these reports, we assumed IgG (RBD) to be one of the indicators for the evaluation of the efficacy of the vaccination and used it to assess factors associated with the low antibody response to the vaccination, in addition to the factors such as advanced age, male gender, and absence of previous infection as mentioned above.

\section{Methods}

\section{Study subjects}

This study was conducted at Tokyo Shinagawa Hospital. The study protocol conformed to the ethical guidelines of the 1975 Declaration of Helsinki and was approved by the Ethics Committee at Tokyo Shinagawa Hospital (approval no. 20-A-34) prior to the start of the study. 655 healthcare workers at Tokyo Shinagawa Hospital including physicians, nurses, physical therapists, medical technologists, and administrative personnel were included in this study after obtaining written informed consent. All the subjects were intramuscularly administered with mRNA COVID-19 vaccine BNT162b2 (Pfizer, NY, USA) twice with three-week interval according to the package insert. Sera were obtained from the subjects prior to the first shot and after two months of the initial shot. The serum samples were subjected to tests for $\lg G(N)$ and $\lg G(R B D)$. The IgG titre measured in sera collected before the vaccination was designated as "pre-vac. IgG" while the IgG titre measured in sera collected two months after the initial vaccination was designated as "post-vac. IgG." Subject information including age, sex, height, weight, history of SARSCoV-2 infection confirmed by PCR, morbidities, and use of immune suppressant during the vaccination period were obtained and anonymized prior to the data analyses. 
We defined the presence of morbidities by the following criteria. The presence of hypertension was defined when a subject was under the treatment, the SBP was more than $139 \mathrm{mmHg}$ or the DBP was more than 89. The presence of diabetes was defined when a subject was under the treatment, had a fasting blood glucose (FBG) level more than $125 \mathrm{mg} / \mathrm{dL}$ or an $\mathrm{HbA} 1 \mathrm{c}$ value more than $6.4 \%$. The presence of dyslipidaemia was defined when a subject was under the treatment, a high-density lipoprotein cholesterol (HDL-C) level less than $40 \mathrm{mg} / \mathrm{dL}$, an LDL-C level more than $139 \mathrm{mg} / \mathrm{dL}$ or a TG level more than $149 \mathrm{mg} / \mathrm{dL}$. The presence of CKD was defined when a subject was under the treatment, had an eGFR less than $60 \mathrm{~mL} / \mathrm{min} / 1.73 \mathrm{~m}^{2}$ or had positive urine protein results. Bronchial asthma and cardiovascular diseases were defined as present when a subject had a history of the disease or were under the treatment. Cancer was defined as present when a subject had a history of the disease or were under the treatment. None of the subjects except for one were under immune suppressant treatment during the study. The one under immune suppressant treatment was designated in Result.

\section{Clinical and laboratory tests}

Real-time RT-PCR test for SARS-CoV-2 was performed on QuantStudio ${ }^{\text {TM }} 5$ Real-Time PCR System (Thermo Fisher SCIENTIFIC, Waltham, MA, USA) which targets N1 and N2 regions of SARS-CoV-2. The PCR result was defined as positive when cycle threshold (Ct) was equal to or less than 40 .

Nasopharyngeal swabs for the PCR were collected by nurses who had ample experience of routine swab collections from fever outpatients and hospitalized patients. Titre of IgG against lgG $(\mathrm{N})$ was measured by an ARCHITECT SARS-CoV-2 IgG assay on Architect i2000 CS5100 (Abbott Laboratories, Abbott Park, IL, USA) and that of IgG (RBD) was measured by an ARCHITECT SARS-CoV-2 IgG II Quant assay on Architect i2000 CS5100 (Abbott Laboratories, Abbott Park, IL, USA). Both assays are based on chemiluminescent microparticle immunoassay (CLIA). According to the package insert of the $\lg G(N)$ assay, cut-off index is $1.4 \mathrm{~S} / \mathrm{C}$ and $\mathrm{CV} \%$ at a mean index of $0.04 \mathrm{~S} / \mathrm{C}$ of 50 negative controls is $5.9 \%$. According to the package insert of the lgG (RBD) assay, cut-off index is $50.0 \mathrm{AU} / \mathrm{mL}$ and lowest concentration at which $\mathrm{CV} \%$ is within $20 \%$ is $7.8 \mathrm{AU} / \mathrm{mL}$.

\section{Statistical analysis}

We used JMP 15.1.0 (SAS Institute Inc., Cary, NC, USA) for univariable and multivariable linear regression analyses. Medians and inter-quartile ranges (IQR's) of continuous subject data including age, BMI, IgG $(\mathrm{N})$, and IgG (RBD) were shown in Table 1 and 3. Categorical data including gender, morbidity, and use of immune suppressant were designated as either 0 (absent) or 1 (present) as shown in Table 2. For the linear regression analyses, IgG $(\mathrm{N})$ and $\lg \mathrm{G}(\mathrm{RBD})$ titres were log-transformed. P-value of $<0.05$ was considered significant.

\section{Results}

\section{Clinical data of the study subjects}


Background of the study subjects including gender, age, body mass index (BMI), morbidities, use of immune suppressant, and positive rates of PCR, IgG against nucleocapsid protein of SARS-CoV-2 (hereinafter designated as "IgG (N)"), and IgG (RBD) were summarized in Table 1. After two months from the initial vaccination, all subjects except for one (99.8\%) who were under immune suppressant treatment showed IgG (RBD) (post-vac. IgG (RBD)) titre of more than $50 \mathrm{AU} / \mathrm{mL}$, a cut-off indicated in the package insert. The post-vac. IgG (RBD) titer of the one under immune suppressant treatment was $43 \mathrm{AU} / \mathrm{mL}$. 600 out of 655 subjects (91.6\%) showed post-vac. IgG (RBD) titre of more than $4,160 \mathrm{AU} / \mathrm{mL}$ and the fifth percentile of post-vac. IgG (RBD) was 3,301.7 AU/mL.

\section{Assessment of factors associated with low IgG (RBD) response to the vaccination}

To assess factors associated with low IgG (RBD) response, we performed univariable and multivariable linear regression analyses as shown in Table 2. By the univariable regression analysis, male gender, advanced age, low titre of pre-vac. IgG $(\mathrm{N})$, use of immune suppressant, and presence of hypertension, dyslipidaemia, and chronic kidney disease (CKD) showed significant association with log-transformed post-vac. IgG (RBD) titre. By the multivariable regression analysis, all these factors remained significant.

As shown in Table 3 and Fig. 1a-d, low titres of post-vac. IgG (RBD) were observed in male gender, subjects with advanced age, low titre of pre-vac. IgG $(\mathrm{N})$, and presence of hypertension, dyslipidaemia, and CKD and use of immune suppressant. One subject who had both hypertension and dyslipidaemia showed post-vac. IgG (RBD) titre of 2,139.7 AU/mL.

\section{Changes of $\lg G(\mathrm{~N})$ during the vaccination period}

To confirm whether IgG $(\mathrm{N})$ titres were not affected by the vaccination, correlation between pre- and postvac. IgG (N) titres was examined (Fig. 2a). Unexpectedly, elevation of $\lg G(\mathrm{~N})$ during the vaccination period was seen in some subjects including four subjects who had been confirmed PCR positive prior to the vaccination. We then examined 23 subjects among the total 655 subjects who had been confirmed PCR positive, and plotted a ratio of pre- vs. post-vac. IgG $(\mathrm{N})$ on the $y$-axis against days post PCR positive (Fig 2b) or against pre-vac. IgG (RBD) (Fig. 2c) on the x-axis. The ratios higher than two were observed around day 100 post PCR positive and pre-vac. IgG (RBD) of less than 2,000 AU/mL.

\section{Discussion}

After the vaccination, the elevation of post-vac. IgG (RBD) above $50 \mathrm{AU} / \mathrm{mL}$ and that of $4,160 \mathrm{AU} / \mathrm{mL}$ were seen in $99.8 \%$ and $91.6 \%$ of the total subjects, respectively. As the percentage of the latter was slightly lower than the one reported by Gobbi et al. (97.4\%) [8], the difference could be due to the difference of elapsed time after the second vaccine shot; ours was five weeks after the second shot while the data of the study by Gobbi et al. were obtained one week after the second shot. In consistent with the report by Gobbi et al. [9], the presence of previous SARS-CoV-2 infection defined in this study by the elevation of pre-vac. IgG $(\mathrm{N})$ above the cut-off $1.4 \mathrm{~S} / \mathrm{C}$ resulted in significantly higher IgG (RBD) titre after the vaccination (Table 2). On the other hand, subjects whose post-vac. IgG (RBD) did not exceed the fifth 
percentile were those who were with pre-vac. IgG (N) below the cut-off (Fig. 1c). This result indicates that repeated immune stimulation, whether by natural infection or vaccination, would help enhance otherwise low antibody response. In fact, the increase of IgG (RBD) was observed even in the subject under the immune suppressant treatment (from $6.9 \mathrm{AU} / \mathrm{mL}$ to $43.0 \mathrm{AU} / \mathrm{mL}$ before and after the vaccination). Albach et al. also reported that repeated vaccination was successful in obtaining a positive antibody response in a patient with rheumatoid arthritis who were under immune suppressant treatment [12].

By the multivariable linear regression analysis shown in Table 2, we confirmed that male gender, subjects with advanced age, and absence of previous infection were significantly associated with low antibody responses, in consistent with the preceding reports $[4,5,8]$. In addition to this, hypertension, dyslipidaemia, CKD, and use of immune suppressant were significantly associated with low antibody responses. The result that the antibody response was low in the subject under the treatment with immune suppressant was theoretically understandable and was actually shown in the report by Albach et al. [12]. Hou et al. suggested that vitamin D deficiency, uremic toxin accumulation and erythropoietin deficiency could affect the immune system in CKD patients [13]. Therefore, the antibody response to the vaccination may have been low in the subjects with CKD (Table 2 and 3 ) due to the compromised immune function.

As Pellini et al. reported that the antibody response to SARS-CoV-2 BNT162b2 vaccine was not associated with hypertension [5], our result showed the statistical significance $(p=0.018)$ on the contrary. In the study by Pellini et al., the geometric means of SARS-CoV-2 S1/S2 IgG with and without hypertension were $172.18 \mathrm{AU} / \mathrm{mL}$ and $307.42 \mathrm{AU} / \mathrm{mL}$, respectively, if not statistically significant. As IgG (RBD) assay used in this study detects IgG directed specifically to the receptor binding domain in SARSCoV-2 S1 subunit, this discrepancy could be due to the different specificity of the IgG assay used in each study.

From the result in Table 2 that showed dyslipidaemia but not BMI was significantly associated with low post-vac. IgG (RBD), it is inferred that the cause for the low antibody response here could be more closely related to regulation of cholesterol rather than obesity in general. Contrary to CKD, hypertension and dyslipidaemia are known to be associated with enhanced autoimmune status $[14,15]$, so that the low antibody response may be caused by a mechanism other than immune modulation in subjects with these diseases. A mechanism related with interaction between $S$ protein and the host receptor could be one of the speculated ones. SARS-CoV-2 is reported to enter host cells in three ways: receptor-mediated plasma membrane fusion, receptor-mediated endocytosis, or antibody-dependent viral entry [16]. For the attachment of SARS-CoV-2 to the host cells in the former two cases, receptor binding domain in S protein binds to its receptor, angiotensin-converting enzyme II (ACE2). It is reported that S protein alone bound to ACE2 and induced several signalling $[17,18]$. It could be inferred, therefore, that some portion of the $S$ protein produced by BNT162b2 binds to ACE2 and endocytosed together with ACE2. As medication for hypertension (e.g., ACE inhibitor, angiotensin receptor blocker) is reported to increase expression of ACE2 [19], highly expressed ACE2 in patients with hypertension may bind and segregate $S$ protein produced by BNT162b2 more efficiently so that reduced amount of $S$ protein remains available for the antigen presentation. By using S2 peptide of SARS-CoV, another subunit of S protein, Meher et al. showed that S2 
peptide was important for the viral entry into the host cell and that the peptide binding status was altered by the membrane cholesterol [20]. Choi et al. suggested that dyslipidaemia might play a role in the severity of COVID-19 infection. [21]. From these reports, it is speculated that the altered status of membrane cholesterol in patients with dyslipidaemia facilitates the entry of S protein into the host cell more efficiently and render the $S$ protein less available for the antigen presentation, just as we postulated for hypertension above. In the subject with both hypertension and dyslipidaemia, post-vac. IgG (RBD) titre was 2,139.7 AU/mL. As shown in Table 3, this titre was lower than the 25th percentile of subject group with hypertension and that with dyslipidaemia, suggesting the additive effect by the two diseases. Whether this is statistically significant, however, remains to be seen.

If we assume that IgG (RBD) titre is correlated with the neutralizing activity to certain extent as mentioned earlier, it would be inferred that the efficacy of the vaccination is weaker in people with hypertension, dyslipidaemia, or CKD as well as those of male gender and of advanced age. If so, we might need to prioritize those people for the additional vaccination. Moreover, the antibody response to the natural infection of SARS-CoV-2 may be attenuated in male gender, people with advanced age, and people with hypertension, dyslipidaemia, or CKD. Li et al. reported that patients who were male, with advanced age, obesity, a history of smoking, hypertension, diabetes, malignancy, coronary heart disease, chronic liver disease, chronic obstructive pulmonary disease (COPD), or CKD were more likely to develop severe COVID-19 symptoms [22]. Takahashi et al. reported that a poor T cell response was correlated with advanced age and was associated with worse disease outcome in male patients [23]. Therefore, the antibody response to the natural infection of SARS-CoV-2 in male gender, people with advanced age, those with hypertension, dyslipidaemia, or CKD may be attenuated and, at least partly, may be more susceptible to the progression of COVID-19.

We unexpectedly saw the elevation of IgG $(\mathrm{N})$ during the vaccination period in four subjects who had been confirmed PCR positive (Fig. 2b), which is interesting because of the similarity with breakthrough infection in those who had been vaccinated with SARS-CoV-2 vaccine reported by Hacisuleyman [24]. The elevation of IgG $(\mathrm{N})$ during the vaccination period could be due to waning immune protection elicited by the previous SARS-CoV-2 infection, breakthrough infection by the variants, or reactivation of persisting virus in the body. Considering that the days from PCR positivity were approximately 100 days in the four subjects (Fig. 2b), it would be too early for the immune response to weaken so that the breakthrough infection of SARS-CoV-2 variants, such as delta which was starting to prevail during that period, is considered to be one of the causes. As shown in Fig. 2c, pre-vac. IgG (RBD) titres in the four subjects were below $2,000 \mathrm{AU} / \mathrm{mL}$, which suggested that the breakthrough infection was not preventable at this antibody level. This is consistent with a report by Bergwerk et al. that the occurrence of breakthrough infections with SARS-CoV-2 was correlated with neutralizing antibody titers during the peri-infection period [25].

By clarifying the antibody level at which SARS-CoV-2 infection could be preventable and by taking factors associated with the low antibody response into account, we would be able to have a more efficient strategy for the vaccination. 


\section{Limitations Of The Study}

This is a single-center-study and the subjects included here are healthcare workers at Tokyo Shinagawa Hospital, so that the subject backgrounds do not necessarily represent those of the other population. Some of the factors examined by the linear regression analyses were of small number (e.g. CKD, immune suppressant), so that the statistical significances could be vulnerable to potential fluctuation. By the multivariable linear regression analysis, the presence of the other unknown factors associated with postvac. IgG (RBD) was indicated, which remains to be seen in the future.

\section{Conclusions}

By using IgG (RBD) assay as an indicator, we confirmed that hypertension, dyslipidaemia, chronic kidney disease, and used of immune suppressant in addition to male gender, advanced age, and absence of previous infection were significantly associated with low antibody response to mRNA COVID-19 vaccine BNT162b2, which we believe would be important information for the planning of the vaccination strategy.

\section{Declarations}

\section{Acknowledgments}

The authors are grateful to those at Tokyo Shinagawa Hospital who were involved in the promotion of this study including healthcare professionals who performed specimen collection and clinical testing, and Clinical Research Coordinators at the study center who were involved in the subject enrollment for this study, notably Ms. Kiyomi Kabasawa who promoted entry of the subject data.

\section{Author Contributions}

S. S., S.O., T. S., M. S., K. K., and M.S. designed the study. M. N., S. O., and M. M. collected and examined the validity of the study data. S. S. and N. I. performed the statistical analyses. S. S., M. N., and S. O. drafted the manuscript. S. S., M. N., S. O., N. I., and M. S. made critical revisions of the manuscript for key intellectual content. All authors reviewed the manuscript before submission.

Competing financial and non-financial interests: The cost of measuring $\lg G(N)$ and $\lg G(R B D)$ was incurred by Abbott Japan. S.S. is an employee of Abbott Japan.M. N., S.O., M. M., T. S., M. S., N. I., K.K., and M. S. have no competing interests. All the authors do not have non-financial competing interests.

\section{References}

1. Polack, F. P. et al. Safety and Efficacy of the BNT162b2 mRNA Covid-19 Vaccine. N. Engl. J. Med, 383, 2603-2615 (2020).

2. Hashimoto, T. et al. High anaphylaxis rates following vaccination with the Pfizer BNT162b2 mRNA vaccine against COVID-19 in Japanese healthcare workers: a secondary analysis of initial post- 
approval safety data. Journal of Travel Medicine, https://doi.org/10.1093/jtm/taab090 (2021).

3. Dagan, N. et al. BNT162b2 mRNA Covid-19 Vaccine in a Nationwide Mass Vaccination Setting. N. Engl. J. Med, 384, 1412-1423 (2021).

4. Naaber, P. et al. Antibody response after COVID-19 mRNA vaccination in relation to age, sex, and side effects. Lancet http://dx.doi.org/10.2139/ssrn.3831845 (2021).

5. Pellini, R. et al. Initial observations on age, gender, BMI and hypertension in antibody responses to SARS-CoV-2 BNT162b2 vaccine. E. Clinical Medicine, 36, 100928 (2021).

6. Liu, J. et al. BNT162b2-elicited neutralization of B.1.617 and other SARS-CoV-2 variants. Nature, https://doi.org/10.1038/s41586-021-03693-y (2021).

7. Davis, C. et al. Reduced neutralisation of the Delta (B.1.617.2) SARS-CoV-2 variant of concern following vaccination. medRxiv. https://doi.org/10.1101/2021.06.23.21259327 (2021).

8. Pfizer, S. Q. 2021 Earnings Teleconference. https://s21.q4cdn.com/317678438/files/doc_financials/2021/q2/Q2-2021-Earnings-ChartsFINAL.pdf (2021).

9. Gobbi, F. et al. Antibody Response to the BNT162b2 mRNA COVID-19 Vaccine in Subjects with Prior SARS-CoV-2 Infection. Viruses, 13, 422 (2021).

10. Garcia-Beltran, W. F. et al. COVID-19-neutralizing antibodies predict disease severity and survival., 184, 476-488 (2021).

11. Muecksch, F. et al. Longitudinal variation in SARS-CoV-2 antibody levels and emergence of viral variants: implications for the ability of serological assays to predict immunity. medRxiv,

PMC8282113, https://doi.org/10.1101/2021.07.02.21259939 (2021).

12. Albach, F. N., Burmester, G. R., Biesen, R. \& Successful BNT162b2 booster vaccinations in a patient with rheumatoid arthritis and initially negative antibody response. $B M J$, https://doi.org/10.1136/annrheumdis-2021-220834 (2021).

13. Hou, Y., Lu, K. \& Kuo, K. The Efficacy of COVID-19 Vaccines in Chronic Kidney Disease and Kidney Transplantation Patients: A Narrative Review. Vaccines, 9, 885 (2021).

14. Rodriguez-Iturbe, B., Pons, H. \& Johnson, R. J. ROLE OF THE IMMUNE SYSTEM IN HYPERTENSION. Physiol. Rev, 97, 1127-1164 (2017).

15. Kim, D. et al. Immunologic Aspects of Dyslipidemia: a Critical Regulator of Adaptive Immunity and Immune Disorders. Journal of Lipid and Atherosclerosis, 10, 184-201 (2021).

16. Zhang, Q. et al. Molecular mechanism of interaction between SARS-CoV-2 and host cells and interventional therapy. Signal Transduction and Targeted Therapy, 6, 233 (2021).

17. Suzuki, Y. J. \& Gychka, S. G. SARS-CoV-2 Spike Protein Elicits Cell Signaling in Human Host Cells: Implications for Possible Consequences of COVID-19 Vaccines. Vaccines, 9, 36 (2021).

18. Lei, Y. et al. SARS-CoV-2 Spike Protein Impairs Endothelial Function via Downregulation of ACE2. Circul. Res, 128, 1323-1326 (2021). 
19. Bosso, M. et al. The Two Faces of ACE2: The Role of ACE2 Receptor and Its Polymorphisms in Hypertension and COVID-19.Molecular Therapy: Methods \& Clinical Development18 (2020).

20. Meher, G., Bhattacharjya, S. \& Chakraborty, H. Membrane Cholesterol Modulates Oligomeric Status and Peptide-Membrane Interaction of Severe Acute Respiratory Syndrome Coronavirus Fusion Peptide. J. Phys. Chem. B, 123, 10654-10662 (2019).

21. Choi, G. J., Kim, H. M. \& Kang, H. The Potential Role of Dyslipidemia in COVID-19 Severity: an Umbrella Review of Systematic Reviews. Journal of Lipid and Atherosclerosis, 3, 435-448 (2020).

22. Li, X. et al. Clinical determinants of the severity of COVID-19: A systematic review and meta-analysis. PLOS ONE, https://doi.org/10.1371/journal.pone.0250602 (2021).

23. Takahashi, T. et al. Sex differences in immune responses that underlie COVID-19 disease outcomes. Nature, 588, 315-320 (2020).

24. Hacisuleyman, E. et al. Vaccine Breakthrough Infections with SARS-CoV-2 Variants. N. Engl. J. Med, 384, 23 (2021).

25. Bergwerk, M. et al. Covid-19 Breakthrough Infections in Vaccinated Health Care Workers. N. Engl. J. Med.10.1056/NEJMoa2109072(2021).

\section{Tables}

Table 1: Clinical data of the study subjects.

BMI, body mass index; COPD, chronic obstructive pulmonary disease; IQR, inter-quartile range. 


\begin{tabular}{|c|c|c|c|c|c|}
\hline & \multirow{2}{*}{$\mathrm{n}$} & \multirow[t]{2}{*}{$\%$} & \multirow[t]{2}{*}{ median (IQR) } & \multirow[t]{2}{*}{$5 \%$ ile } \\
\hline Subjec & mber & & & & \\
\hline $\begin{array}{l}\text { Male } \\
\text { gender }\end{array}$ & & 194 & $29.6 \%$ & & \\
\hline Age & & & & $31.0(25.0-43.8)$ & \\
\hline BMI & $\mathrm{kg} / \mathrm{m}^{2}$ & & & $21.5(19.7-23.9)$ & \\
\hline \multicolumn{6}{|c|}{$\begin{array}{l}\text { Morbidit } \\
\text { ies }\end{array}$} \\
\hline & Hypertension & 12 & $1.8 \%$ & & \\
\hline & Diabetes & 2 & $0.3 \%$ & & \\
\hline & Dyslipidemia & 7 & $1.1 \%$ & & \\
\hline & Bronchial asthma & 20 & $3.1 \%$ & & \\
\hline & COPD & 0 & $0.0 \%$ & & \\
\hline & Cardiovascular disease & 2 & $0.3 \%$ & & \\
\hline & Chronic kidney disease & 1 & $0.2 \%$ & & \\
\hline & Cancer & 3 & $0.5 \%$ & & \\
\hline With i & une suppressant & 1 & $0.2 \%$ & & \\
\hline Positiv & te pre-vaccination & & positive & & \\
\hline & PCR & 23 & $3.5 \%$ & & \\
\hline & $\operatorname{IgG}(\mathrm{N})$, cut-off $=1.4 \mathrm{~S} / \mathrm{C}$ & 39 & $6.0 \%$ & $0.07(0.03-0.22)$ & \\
\hline & $\begin{array}{l}\text { IgG (RBD), cut-off }=50 \\
\mathrm{AU} / \mathrm{mL}\end{array}$ & 60 & $9.2 \%$ & $4.6(2.7-7.8)$ & \\
\hline Positiv & te post-vaccination & & ositive & & \\
\hline & $\operatorname{IgG}(N)$, cut-off $=1.4 \mathrm{~S} / \mathrm{C}$ & 37 & $5.6 \%$ & $0.09(0.03-0.24)$ & \\
\hline & $\begin{array}{l}\text { IgG }(\mathrm{RBD}), \text { cut-off }=50 \\
\mathrm{AU} / \mathrm{mL}\end{array}$ & 654 & $99.8 \%$ & $\begin{array}{c}12,294.0(7,483.0- \\
19,153.2)\end{array}$ & $\begin{array}{l}3301.7 \\
\mathrm{AU} / \mathrm{mL}\end{array}$ \\
\hline
\end{tabular}

Table 2: Univariable and multivariable linear regression analyses of subject background against log (IgG (RBD) post-vaccination). HT, hypertension; DM, diabetes mellitus; DL, dyslipidaemia; BA, bronchial asthma; CVD, cardiovascular disease; CKD, chronic kidney disease; Im sup, immune suppressant; SE, standard error. 
Univariable linear regression

\begin{tabular}{|c|c|c|c|c|c|c|c|c|}
\hline & \multicolumn{4}{|c|}{ Girvallavie mical legression } & \multicolumn{4}{|c|}{ 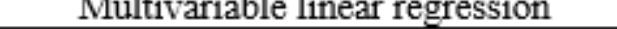 } \\
\hline & $\begin{array}{c}\text { Coeffici } \\
\text { ent }\end{array}$ & $\mathrm{SE}$ & $\mathrm{t}$ & p-value & $\begin{array}{c}\text { Coeffici } \\
\text { ent }\end{array}$ & $\mathrm{SE}$ & $\mathrm{t}$ & p-value \\
\hline Intercept & & & & & 4.4 & 0.0 & 114.2 & $<0.001$ \\
\hline Gender $(f=0, m=1)$ & -0.1 & 0.0 & -5.4 & $<0.001$ & -0.1 & 0.0 & -4.8 & $<0.001$ \\
\hline Age & 0.0 & 0.0 & -8.0 & $<0.001$ & 0.0 & 0.0 & -6.5 & $<0.001$ \\
\hline Body Mass Index & 0.0 & 0.0 & -1.5 & 0.130 & & & & \\
\hline $\begin{array}{l}\log (\operatorname{IgG}(\mathrm{N}) \text { pre- } \\
\text { vac.) }\end{array}$ & 0.1 & 0.0 & 3.7 & $<0.001$ & 0.1 & 0.0 & 3.0 & 0.003 \\
\hline HT $($ no= $=0$, yes=1) & -0.4 & 0.1 & -4.1 & $<0.001$ & -0.2 & 0.1 & -2.4 & 0.018 \\
\hline $\mathrm{DM}(\mathrm{no}=0$, yes $=1)$ & -0.2 & 0.2 & -0.7 & 0.490 & & & & \\
\hline $\mathrm{DL}(\mathrm{no}=0$, yes $=1)$ & -0.5 & 0.1 & -3.8 & $<0.001$ & -0.3 & 0.1 & -2.5 & 0.014 \\
\hline $\mathrm{BA}($ no $=0$, yes $=1)$ & 0.0 & 0.1 & 0.2 & 0.877 & & & & \\
\hline $\operatorname{CVD}($ no $=0$, yes $=1)$ & 0.0 & 0.2 & 0.2 & 0.858 & & & & \\
\hline $\mathrm{CKD}(\mathrm{no}=0$, yes $=1)$ & -1.3 & 0.3 & -3.9 & $<0.001$ & -1.1 & 0.3 & -3.7 & $<0.001$ \\
\hline Cancer $($ no $=0$, yes $=1)$ & -0.3 & 0.2 & -1.4 & 0.168 & & & & \\
\hline Im sup (no $=0$, yes $=1)$ & -2.5 & 0.3 & -7.9 & $<0.001$ & -2.3 & 0.3 & -8.0 & $<0.001$ \\
\hline
\end{tabular}

Table 3: IgG (RBD) titre two month after the vaccination

$H T$, hypertension; $D L$, dyslipidaemia; $C K D$, chronic kidney disease; IQR, inter-quartile range.

\begin{tabular}{llc} 
& & AU $/ \mathrm{mL}$, median (IQR) \\
\hline Gender & Memale & $13711.6(8560.2,20022.3)$ \\
& & $10136.6(5651.1,16863.8)$ \\
Age group & $20-29$ years old & \\
& $30-39$ years old & $14525.6(9700.9,21073.0)$ \\
& $40-49$ years old & $13291.7(7488.7,20061.6)$ \\
& $50-59$ years old & $10426.2(6576.0,15374.4)$ \\
& $>=60$ years old & $9618.8(6220.5,14036.9)$ \\
& & $6753.6(3944.5,11216.9)$ \\
IgG (N) pre-vac. & $<1.4$ S/C & \\
& $>=1.4$ S/C & $11970.4(7409.6,18319.8)$ \\
Morbidity and treatment & $19614.9(13064.4,30361.8)$ \\
& Total subjects & \\
& HT & $12294.0(7483.0,19153.2)$ \\
& DL & $4415.3(3916.8,6813.9)$ \\
& CKD & $3329.9(2663.4,7226.2)$ \\
& With immune suppressant & $604.3(\mathrm{~N} . \mathrm{A})$. \\
& & $43.0(\mathrm{~N} . \mathrm{A})$. \\
\hline
\end{tabular}

\section{Figures}



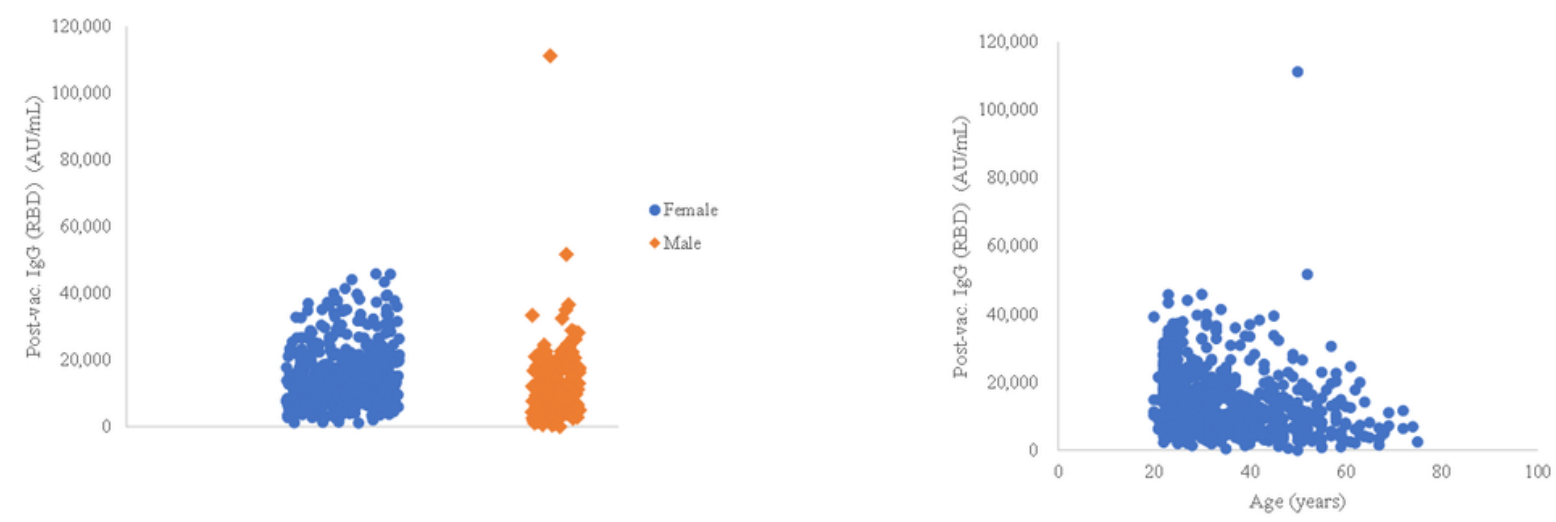

Fig. 1c

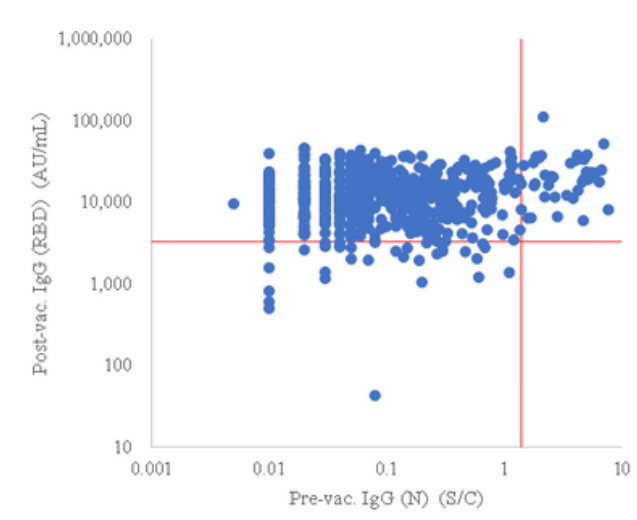

Fig. 1d

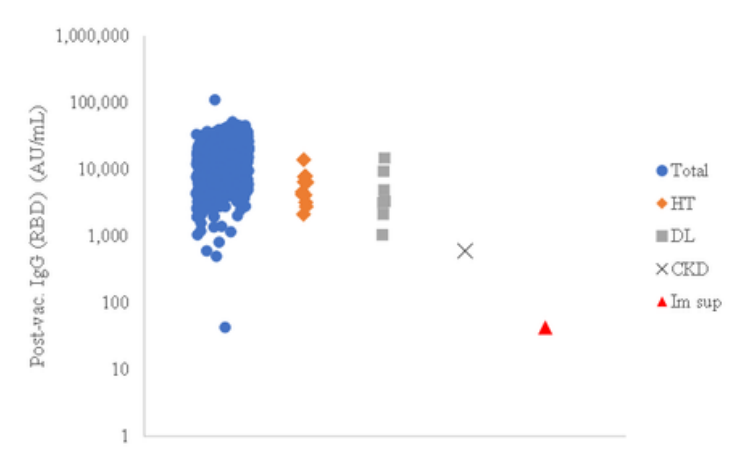

\section{Figure 1}

Distribution of post-vac. IgG (RBD) by gender (Fig. 1a), age (Fig. 1b), previous SARS-CoV-2 infection indicated by pre-vac. IgG (N) titer (Fig. 1C), and morbidities and use of immune suppressant (Fig. 1d). In Fig. 1c, the red line on the x-axis represents the cut-off of $\operatorname{lgG}(\mathrm{N}), 1.4 \mathrm{~S} / \mathrm{C}$, and the red line on the $y$-axis represents the fifth percentile of post-vac. IgG (RBD), 3,301.7 AU/mL. HT, hypertension; DM, diabetes mellitus; DL, dyslipidaemia; CKD, chronic kidney disease; Im sup, immune suppressant. 
Fig. 2a
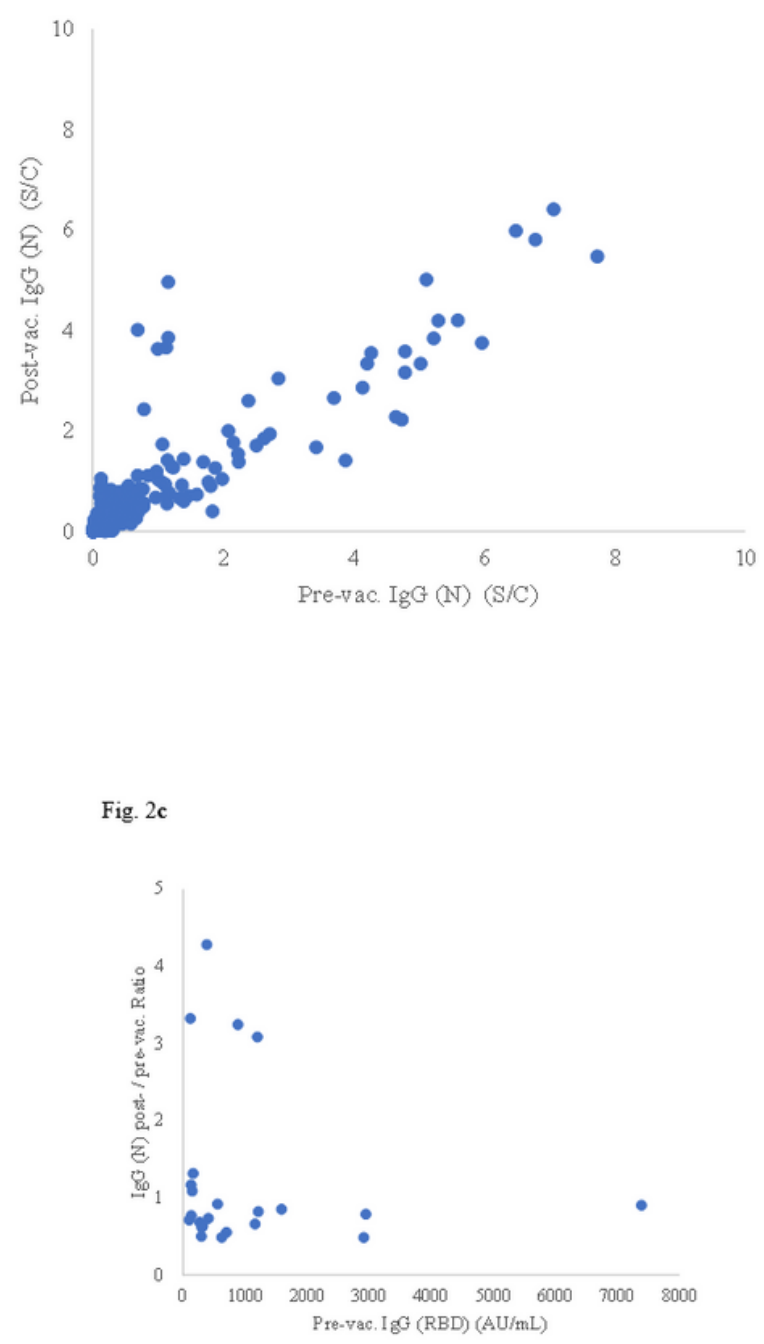

Fig. 2b

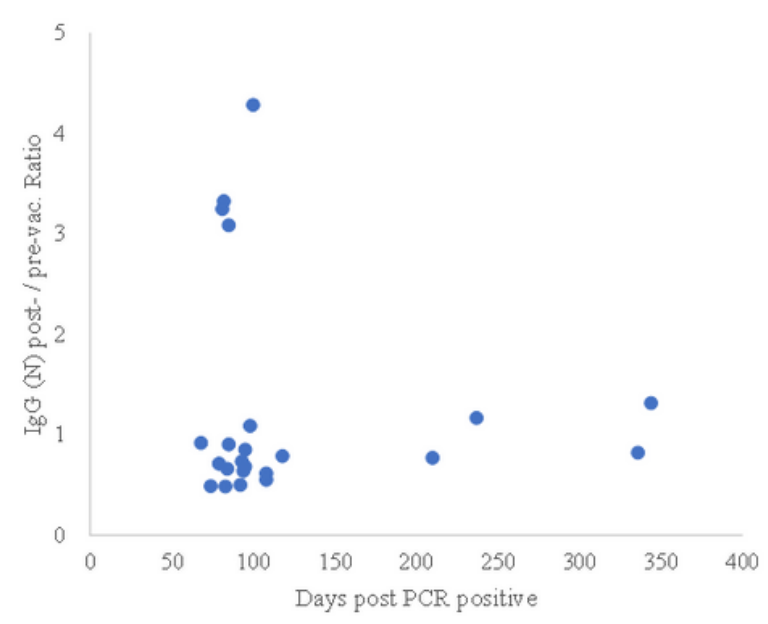

Figure 2

Changes of $\operatorname{lgG}(\mathrm{N})$ from pre to post-vaccination. Fig. 2a: correlation between pre-vac. IgG $(\mathrm{N})$ (x-axis) and post-vac. IgG $(\mathrm{N})$ (y-axis) in total subjects $(\mathrm{n}=655)$. Fig. 2b: Ratio of post- vs. pre-vac. IgG ( $\mathrm{N})(\mathrm{y}$-axis) against days post PCR positive in subjects with confirmed PCR positivity $(n=23)$. Fig. 2c: Ratio of postvs. pre-vaccination IgG $(\mathrm{N})$ (y-axis) against pre-vac. IgG $(\mathrm{RBD})$ in subjects with confirmed PCR positivity $(n=23)$. 